\title{
Effects of Physician Communication and Family Hardiness on Patient Medication Regimen Beliefs and Adherence
}

Todd D Molfenter* and Roger L Brown

Center for Health Enhancement Systems Studies (CHESS), Mechanical Engineering Building, University Avenue Madison, USA

\begin{abstract}
This study aims to develop a model that describes how physician communication and family hardiness affect medication regimen beliefs and adherence for patients on regimens to control diabetes and hyperlipidemia (high cholesterol). Study participants $(n=1356)$ completed self-report questionnaires on health beliefs. Pharmacy refill records from a health plan in the United States provided data on their medication adherence. Structural equation modeling was used to model variable relationships. A mediation analysis demonstrated that physician communication behaviors had a significant impact on the patients' behavioral intention to adhere to their regimen and medication adherence when they were mediated by the patient's medication taking health beliefs related to perceived benefit of the regimen, impact of side effects, and cost of regimen. Conversely, family hardiness had no effect on medication-taking behaviors. To improve patient medication-taking behaviors, physician communication behaviors should be targeted. The study suggests the physician's initial regimen discussion is important to both regimen initiation and long-term adherence, and should emphasize the regimen's benefits and how to avoid common side effects. Also, establishing a follow-up physician-patient relationship can enhance regimen adherence and reduce the likelihood that a patient will stop taking the medication due to cost concerns. The research supports the important role the physician plays in health behavior maintenance. Future research should study the effect physicians have on other recurring health behaviors.
\end{abstract}

Keywords: Medication adherence; Physician-patient communication; Family hardiness

\section{Introduction}

Medication therapy is vital for treatment of chronic diseases, but non-adherence to medication is a primary reason for treatment failure in diseases such as heart disease and diabetes [1-3]. Untreated heart disease is the leading cause of mortality in the United States (U.S.) [4]. Diabetes is the leading cause of kidney disease, blindness, and lower limb amputation among adults, aged 20-74 [5]. For heart disease, statin medications reduce major vascular events by 20 percent [6]. Yet, just 40-60 percent of those on a statin regimen adhere to it sufficiently to experience the benefits of the treatment $[7,8]$. For diabetes, the clinical impact of many simple and effective diabetic therapies has been limited by poor adherence rates [9]. Despite the medical community's increased reliance on medical therapy to treat disease, medication adherence rates have remained relatively unchanged for 40 years [10-12]. As a result, non-adherent patients have poorer clinical outcomes and higher medical costs than medication-adherent patients $[3,13]$.

Understanding and predicting medication adherence is complicated by the diverse set of factors affecting this behavior [14]. The roles of the patient's physician and family have received considerable attention in medication adherence research $[15,16]$. Medication adherence interventions tend to take place in the physician's office or the patient's home because of the role these settings play in a patient's health care maintenance and daily living [17].

\section{Two social factors of medication adherence}

Clinician-patient communication is considered an important and modifiable determinant of patient adherence behaviors. The physician communication literature has found that positive physician communication patterns result in 1.64 times higher patient adherence [16]. Positive physician communication patterns are enhanced during the initial regimen discussion by using patient-centered counseling that proactively engages the patient [18]. When the patient believes that there is concordance between their preferences and the physician prescribed regimen, greater medication adherence rates result [19]. Podl et al. [20] also found that the physician's communication style is important during both the initial regimen discussion and follow-up appointments. These communications offer opportunities to sustain individual motivation, assess progress, provide feedback, and adjust behavior plans. Providers who develop "relationships" that foster continuity, trust, and accountability tend to have more adherent patients [21]. It is less clear; however, which aspects of the provider's communications patterns are most critical for maintaining medication adherence behaviors and how these patterns influence patient health beliefs [22]. A patient's health beliefs are considered to be an important antecedent to the intention to perform and actually engage in a health behavior [23,24]. Patient health beliefs are directly related to medication adherence behavior [25]. Investigating the causal chain that occurs between provider communication patterns, the development of patient health beliefs, and medication adherence could provide insights into how patients arrive at medication adherence decisions [26]. In practice, understanding which of these pathways are most relevant to medication adherence behavior could guide physicians in how to design meaningful communication patterns to enhance medication adherence.

The effectiveness of physician-patient communication may not be an isolated activity and could be affected by the environment patients will return to following their physician visits. Family environment does impact medication adherence [27]. Medication adherence is 1.74 times higher in cohesive families and 1.53 times lower in families with conflict [15]. Several theories suggest that family resilience or hardiness can help individual members cope with stressors in the environment,

*Corresponding author: Todd D Molfenter, Center for Health Enhancement Systems Studies (CHESS), 4103 Mechanical Engineering Building, 1513 University Avenue, Madison, WI 53706, USA, Tel: (608) 262-1685; Fax: (608) 8901438; E-mail: todd.molfenter@chess.wisc.edu

Received November 22, 2013; Accepted March 25, 2014; Published April 30 2014

Citation: Molfenter TD, Brown RL (2014) Effects of Physician Communication and Family Hardiness on Patient Medication Regimen Beliefs and Adherence. Gen Med (Los Angel) 2: 136. doi: 10.4172/2327-5146.1000136

Copyright: (c) 2014 Molfenter TD, et al. This is an open-access article distributed under the terms of the Creative Commons Attribution License, which permits unrestricted use, distribution, and reproduction in any medium, provided the original author and source are credited. 